\title{
GCU
}

Glasgow Caledonian

University

University for the Common Good

\section{Inpatient hospital burden of hepatitis C-diagnosed patients with decompensated cirrhosis}

McDonald, Scott A.; Innes, Hamish; Aspinall, Esther J.; Hayes, Peter C. ; Alavi, Maryam ; Valerio, Heather; Goldberg, David J.; Hutchinson, Sharon J.

Published in:

Liver International

DOI:

10.1111/liv.13681

Publication date:

2018

Document Version

Author accepted manuscript

Link to publication in ResearchOnline

Citation for published version (Harvard):

McDonald, SA, Innes, H, Aspinall, EJ, Hayes, PC, Alavi, M, Valerio, H, Goldberg, DJ \& Hutchinson, SJ 2018, 'Inpatient hospital burden of hepatitis C-diagnosed patients with decompensated cirrhosis', Liver International, vol. 38, no. 8, pp. 1402-1410. https://doi.org/10.1111/liv.13681

\section{General rights}

Copyright and moral rights for the publications made accessible in the public portal are retained by the authors and/or other copyright owners and it is a condition of accessing publications that users recognise and abide by the legal requirements associated with these rights.

Take down policy

If you believe that this document breaches copyright please view our takedown policy at https://edshare.gcu.ac.uk/id/eprint/5179 for details of how to contact us. 
Inpatient hospital burden of hepatitis C-diagnosed patients with decompensated cirrhosis

Scott A McDonald ${ }^{1,2}$, Hamish A Innes ${ }^{1,2}$, Esther Aspinall ${ }^{1,2}$, Peter C Hayes ${ }^{3}$, Maryam Alavi², Heather Valerio ${ }^{1,2}$, David J Goldberg ${ }^{1,2}$, Sharon J Hutchinson ${ }^{1,2}$

${ }^{1}$ School of Health and Life Sciences, Glasgow Caledonian University, Scotland, UK

${ }^{2}$ Health Protection Scotland, Glasgow, Scotland, UK

${ }^{3}$ Royal Infirmary of Edinburgh, Edinburgh, Scotland, UK

Corresponding author:

Dr Scott A McDonald

Glasgow Caledonian University

School of Health and Life Sciences

Cowcaddens Road

Glasgow G4 0BA, Scotland, UK

Email: smcdonald4@nhs.net

Word count: 4998

Figure/tables: 5

Abbreviations:

HCV: Hepatitis C virus

DC: Decompensated cirrhosis

DAA: Direct acting antiviral

HCC: Hepatocellular carcinoma

ICD: International Classification of Diseases

Conflicts of interest: HAI reports personal fees from Janssen and speaker fees from Gilead, outside the submitted work. SJH has received honoraria for presenting at meetings/conferences from Abbvie, Gilead, Janssen, MSD, Roche.

Financial support: None declared 


\section{ABSTRACT}

Background \& Aims: To describe the burden on inpatient hospital resources over time from patients diagnosed with hepatitis $\mathrm{C}$ virus $(\mathrm{HCV})$ infection and who have reached the decompensated stage of cirrhosis (DC), as existing estimates of hospital stay in these patients are limited.

Methods: A retrospective longitudinal dataset was formed via record-linkage between the national HCV diagnosis database and inpatient/daycase hospitalisation and death registers in Scotland. The study population consisted of HCV-diagnosed patients with a first DC admission in 1996-2013, with follow-up available until 31 May 2014. We investigated and quantified the mean cumulative length of hospital stay, distributions over discharge diagnosis categories, and trends in admission rates.

Results: Among our study population ( $n=1543)$, we identified 10,179 admissions with any diagnosis post-first DC admission. Between 1996 and 2013 there was a 16-fold rise in annual total admissions (from 112 to 1791) and an 11-fold rise in hospital stay (719 to 8045). When restricting minimum possible follow-up to two years, DC patients $(n=1312)$ had an overall admission rate of 7.3 per person-year, and spent on average 43 days (26 days during first 6 months) in hospital; for all liver-related, liver-related other than HCC/DC, and non-liver related only admissions, this was 39,14 , and 5 days, respectively.

Conclusions: HCV-infected DC patients impose a considerable inpatient hospital burden, mostly from DC- and other liver-related admissions, but also from admissions associated with non-liver co-morbidities. Estimates will be useful for monitoring the impact of prevention and treatment, and for computing the cost-effectiveness of new therapies. 
Keywords: Hospital stay; Decompensated cirrhosis; Hepatitis C virus; Scotland

\section{KEYPOINTS}

- Patients with HCV who have progressed to decompensated liver disease represent a substantial burden on health care resources.

- Up-to-date figures on resource use, such as hospital stay, based on a large patient sample, are required to inform cost-effectiveness models of current DAA treatments.

- We provide estimates of the morbidity burden in HCV-infected DC patients, for both liver disease and also non-liver co-morbidities and behaviours, which contributed notably to the inpatient hospital burden.

- These findings constitute a baseline against which any improvements in the care and management of DC patients can be evaluated upon wider availability of DAA therapies. 


\section{INTRODUCTION}

Chronic infection with the hepatitis $\mathrm{C}$ virus $(\mathrm{HCV})$ can lead to the generally asymptomatic phase of compensated cirrhosis, from which progression to the severe, life-threatening stage of decompensated cirrhosis (DC) occurs with a 10 -year cumulative incidence of $42 \% .{ }^{1}$ In the predirect acting antiviral (DAA) therapy era, the annual risk of mortality following DC without liver transplantation was very high. ${ }^{2}$ A recent Scottish record-linkage study reported a 5-year cumulative risk of mortality of $70 \%$ after first inpatient hospital admission for DC, with no evidence for any improvement in mortality risk over the 20-year study period 1994-2013. ${ }^{3}$ Upon expansion of DAA treatment to DC-stage patients, ${ }^{4}$ promising reported viral clearance rates ${ }^{5,6}$ may translate to improved survival, but data from long-term follow-up studies are not yet available.

Mortality findings may not generalise to temporal trends in the morbidity burden. Because of - in some cases, extensive - co-morbidities among HCV-infected persons (for instance, mental health disorders, ${ }^{7}$ or conditions related to lifestyle behaviours such as problem alcohol/drug use; ${ }^{8}$ or chronic diseases prevalent in older persons: cardiovascular and respiratory impairments; diabetes, cancer; e.g., Ref. 9), it is important to quantify the health-care associated burden in this patient population attributable to both liver- and non-liver related causes.

The financial burden due to patient care and management once chronic HCV patients progress to the DC stage is recognised as considerable. For instance, economic evaluations carried out for the USA birth cohort screening initiative adopted annualised direct costs for DC patient care in the range of US\$11,000 to $\$ 28,000 .^{10-12}$ In the European setting, annual hospitalisation costs of 8000 20,000 euros for DC patients have been estimated for France ${ }^{13}$ and 28,000 euros for the Netherlands. ${ }^{14}$ 
In one of the few UK studies to quantify the length of hospital stay in HCV-infected patients according to disease stage, ${ }^{15} 21.1$ days was reported as the mean annualised length of stay in hospital for DC patients (without HCC), with 22.6 days per year for HCC patients (with or without DC). The authors translate these figures to a mean annual total cost of $£ 9,120$ per year (in 2002/03 prices), of which the principal cost component was inpatient hospital stay, accounting for more than $70 \%$ of the total cost. These values are based on $40 \mathrm{DC}$ and $20 \mathrm{HCC}$ patients taking part in an observational costing substudy of the UK Mild Hepatitis C trial, ${ }^{15,16}$ and the associated costeffectiveness analysis for the National Institute for Health and Care Excellence depended on these burden estimates. Although estimates from the MILD trial are derived from a small study population over a two-year recruitment period more than 15 years ago, subsequent economic analyses both in and outwith the UK still use these figures (e.g., Refs. 17,18). It is therefore prudent to derive updated estimates using a larger, national sample of patients, and to consider the excess hospitalisation that may not be directly related to HCV liver disease, but nonetheless occurs in these patients. Updated hospital stay data will also serve a vital role for assessing the impact of interferon-free antiviral therapy in preventing DC and its associated morbidity.

The current study follows-up a large nationwide cohort of patients admitted to hospital with DC, to generate real-world estimates of morbidity burden in this population. Our primary goal was to quantify the extent of inpatient hospital usage among DC patients by calculating the mean cumulative length of stay for all admissions and for liver-related and non-liver related admission categories separately. As secondary objectives we analysed temporal trends in admission rates, and investigated the distribution of admissions according to severity over the follow-up period. 


\section{MATERIALS AND METHODS}

\section{Data sources and record-linkage}

Linkage of three national data sources was carried out by Information Services Division (a division of NHS National Services Scotland) using probabilistic record-linkage techniques. ${ }^{19}$ This linked dataset was anonymized prior to transfer to HPS for analysis. Data linkages were approved by the Privacy Advisory Committee, which oversees confidentiality issues involving data held on NHS Scotland patients. Further ethical approval and informed consent were not required for this database study.

The linked dataset consisted of: (i) a database, maintained by Health Protection Scotland, of all persons in Scotland who have been diagnosed HCV positive since testing commenced in 1991 (including stored serum back to 1985); ${ }^{20}$ laboratory detection of hepatitis C antibody is an inclusion criterion. As of 31 December 2013, the HCV Diagnosis database contained records for 35,474 persons. ${ }^{21}$ It is estimated that approximately $55 \%$ of the current $\mathrm{HCV}$-infected Scottish population have been diagnosed..$^{22}$ Source (ii) is the Scottish Morbidity Records, an episode-based patient record held by Information Services Division of all general acute inpatient and day-case hospital discharges. Discharge diagnoses use International Classification of Diseases (ICD) Tenth Revision for hospital discharges in the period 1996-2014. Data source (iii) is the national death registry held by the National Records of Scotland (NRS). Underlying and contributing causes of death are coded using ICD-9 and ICD-10 for deaths in 1996-1999 and 2000-2014, respectively. We had deaths and hospitalisation data available until 31 May 2014, and began our analysis in 1 January 1996, the year when ICD-10 was implemented in the Scottish Morbidity Records.

A further linkage between the HCV Diagnosis and the Scottish Hepatitis C Clinical Database was 
conducted at HPS to determine the HCV RNA status at date of index DC admission for those patients who had previously been successfully treated with antiviral therapy (see Ref. 23 for further details). This database, also held at Health Protection Scotland, contains data on all aspects of HCV care and patient management and is deployed at specialist HCV treatment centres within Scotland.

\section{Study population}

The study population consisted of all HCV-diagnosed patients with a first inpatient/day-case admission for DC in Scotland over the period 1996-2013. Patients whose first DC admission occurred more than one year preceding HCV diagnosis were excluded, in an attempt to reduce potential bias due to DC arising from a different aetiology. HCV RNA status was not restricted to PCR-positive in the principal analysis, but in sensitivity analyses we stratify by RNA status.

An admission for DC was defined according to the presence of a relevant ICD-10 code(s) in either the main or five supplementary diagnosis fields of the episode record. DC discharge diagnosis codes were categorised into: hepatic failure (aggregating the diagnosis codes comprising chronic hepatic failure, alcoholic hepatic failure, and hepatorenal syndrome), ascites, and bleeding oesophageal varices (for ICD-10 codes comprising these definitions see Supporting Information, Table S1). The selected diagnosis codes have been used in previous DC research. ${ }^{3,24}$ We use the terms admission and discharge equivalently, assuming that discharge diagnoses encode the reason for admission. Successive inpatient episodes without release from hospital, and/or transfers between specialty or consultant, with identical sets of discharge diagnoses, are counted as a single admission. Episodes with an identical date of admission and discharge (most often day-cases) contributed 0.5 days to measures of total hospital stay. 


\section{Patient characteristics and exposure variables}

Demographic and behavioural factors (sex, age, risk group for acquiring HCV infection, history of an alcohol-related hospitalisation prior to first DC admission), HCV-related factors (RNA status at first DC admission, operationalised as the most recent PCR test result: positive, negative, or not known; relevant for sensitivity analysis), and liver disease-related variables (prevalence of cirrhotic complications - based on discharge diagnosis codes - at index DC admission, history of HCC admission(s) prior to first DC admission), and mortality data were extracted and tabulated.

\section{Data analysis}

Analysis goals. Our specific aims were to: (i) estimate the burden of hospital usage by DC patients as mean cumulative length of stay, both overall and stratified by admission diagnosis category, and determine the factors associated with cumulative length of stay; (ii) describe the inpatient hospital burden according to time since first DC admission; (iii) investigate temporal trends in the annual total admissions and admission rate, and estimate the associations between admission rate and patient factors; and (iv) summarise the distribution over severity-ranked categories of hospital admission and compute mean length of stay as a function of follow-up time, distinguishing liverand non-liver related admissions.

Outcome measures. From the DC patients' entire hospital record including and subsequent to the first DC admission, the total number of inpatient admissions, admission rate, and total stay in hospital were calculated for each year of the study period, and the mean cumulative stay per patient was calculated according to various follow-up definitions (see below). We report all-cause admissions (i.e., with any diagnosis), and distinguish liver-related and non-liver-related 
admissions: (a) liver-related, consisting of: HCC, hepatic failure (chronic liver failure, hepatorenal syndrome), ascites/bleeding varices, other liver-related admissions (which includes alcohol-related diagnosis codes; see Table S1); (b) non-liver related admissions - these consisted of all other admissions in which none of the above codes were indicated in any of the five discharge diagnosis fields. The subcategories of liver-related admissions were defined hierarchically according to severity (itself defined according to short-term mortality risk); i.e., if an HCC code occurred in any diagnosis field, then the episode was categorised as HCC. However, if no HCC code, but a hepatic failure code occurred in any diagnosis field, then the episode was classified as hepatic failure. Classification of episodes into categories lower in the hierarchy was conducted analogously; i.e., if no liver-related code occurred in any diagnosis field, the episode was classified as a non-liver related admission.

Within the category 'non-liver related', four subcategories were defined based on frequency of occurrence: circulatory and respiratory, digestive system other than liver, injuries and poisonings, and other non-liver (Supporting Information, Table S1). Each non-liver related episode was classified using an analogous hierarchical procedure.

Follow-up. Two definitions of follow-up were used, specific to the type of analysis. In the first (for producing Table 2), the study population was defined as those HCV-diagnosed persons with a first DC admission between 1996 and 31 May 2012; this ensured a minimum potential observation period of two years for all patients. Follow-up was censored at (i) 31 May 2014, regardless of patients' date of first DC admission, and also according to three censoring thresholds: (ii) 2 years, (iii) 1 year, and (iv) 6 months following index admission. 
In the second follow-up definition, in which admission rate across triennium of the study period is investigated (Table 1b), follow-up was defined to begin at the date of the first DC hospital admission, and to end at five months following the end of the triennium in which the first DC admission fell. For example, for the last triennium (2011-2013), follow-up was censored at 31 May 2014; similarly, follow-up for the triennium 2008-2010 was censored at 31 May 2011. This was to ensure comparability in admission rates across period of first DC admission, by equalising the maximum possible amount of follow-up time. For both definitions, follow-up was also censored at date of death, if occurred.

Sensitivity analyses. We conducted comparable analyses to those described earlier, but restricted the study population to HCV RNA-positive persons, and also compared mean cumulative length of stay across HCV RNA status categories (see Supporting Information, Tables S2 and S3).

Statistical analyses. Multifactorial Poisson regression was used to infer the patient and other factors (age, sex, RNA status, admission for HCC prior to first DC admission, period of first DC admission) associated with cumulative length of hospital stay. We define cumulative length of stay as the total stay in hospital (i.e., summing multiple admissions) within a patient's follow-up period. We used Cox regression with robust standard errors to confirm temporal trends in admission rates for all admissions, and in admission rates categorised as liver-related only, controlling for important confounders (age, sex, risk group, prior alcohol-related admission, HCV RNA status, and prior HCC admission).

The counting-process formulation for recurrent events was adopted for person-time analyses. All statistical analyses were conducted using R statistical computing software. ${ }^{25}$ 


\section{RESULTS}

\section{Patient characteristics}

A total of 1,543 HCV diagnosed persons had a first inpatient hospital admission for DC within the period 1996-2013, increasing 3.9-fold between 1996-98 and 2011-13 (Table 1a). Males constituted the majority of patients (73\%), and the mean age at first DC admission was 47 years. Previous to their first DC admission, $56 \%$ and $4.2 \%$ had an alcohol-related admission or HCC admission, respectively. Seventy-seven percent of patients had tested HCV RNA-positive prior to index admission (85\% of those whose HCV RNA status was known). As of 31 May 2014, 457 (30\%) DC patients were not known to have died.

The most prevalent DC diagnosis code at index DC admission, over all six triennia, was ascites (57\%), followed by chronic hepatic failure (21\%). The prevalence of both ascites and alcoholic hepatic failure tend to increase with triennium, whereas the prevalence of bleeding varices tend to decrease over the study period.

\section{Temporal trends in inpatient admissions and total stay}

Between 1996 and 2013, the total number of inpatient admissions among the study population of HCV diagnosed patients with DC increased 16-fold, from 112 to 1791, and the annual total inpatient hospital stay also increased 11-fold over the same period, from 719 days in 1996 to 8045 in 2013 (Fig. 1), reflecting rising DC patient numbers. The distribution over admission category in the year 2013 was HCC/DC (4382 days), other liver-related (2482 days), and non-liver related only (1180 days). 
Aggregating over all six triennia, there were 10,179 inpatient admissions in total, for an overall admission rate of 7.9 admissions per person-year (Table $1 \mathrm{~b}$ ). When computed separately by triennium of first DC admission, there was an apparent increasing trend in admission rate from 1999-2001 (7.4 per person-year) to 2011-2013 (9.6 per person-year). Cox regression analysis, adjusting for confounders, confirmed this increasing trend for all admission categories (HR for year of first DC admission=1.024, 95\% CI: 1.019-1.029) and for liver-related admissions only (HR for year $=1.023,95 \%$ CI: 1.017-1.029) (see Supporting Information, Table S4). The median length of stay per admission was constant at 3 days from the beginning of the study period, dropping to 2 days in the most recent triennium.

In sensitivity analysis of the study population restricted to chronically-infected (HCV RNApositive) individuals, very similar temporal trends in admission rates were apparent (Supporting Information, Table S3).

\section{Length of stay since first DC admission}

Considering all admissions as a function of time since index DC admission, there was a rapid drop off in the proportion of patients in hospital, with time. The mean cumulative length of stay per patient stratified by admission code category, according to three censored follow-up period definitions, is shown in Table 2. The study population for this analysis consisted of 1312 patients with a first admission between 1 January 1996 and 31 May 2012. Considering 2 years of follow-up starting with the index admission, the mean cumulative length of stay per patient was 43 days, with averages of 39 and 5 days for liver- and non-liver related only coded admissions, respectively. Shorter (i.e., 1 year and 6 months) censoring thresholds gave disproportionately lower average cumulative lengths of stay, illustrating the greater chance of subsequent hospitalisation following 
index admission. Among all admissions, $79 \%$ and $60 \%$ of the mean cumulative stay calculated over the first 2 years of follow-up occurred within the first year and the initial 6 months, respectively.

Patient factors associated with cumulative length of inpatient stay were determined via Poisson regression (Table 3). Patients aged 60+ years had longer total stay (RR=1.11, 95\% CI: $1.08-1.14$ ), as did patients with one or more alcohol-related admissions before first DC admission ( $R R=1.21,95 \%$ CI: 1.19-1.23), but having ever been admitted for HCC prior to first DC admission ( $R R=0.68,95 \%$ CI: $0.64-0.71)$ or if first DC admission was for bleeding varices ( $R R=0.83,95 \%$ CI: $0.80-0.85)$ was associated with a shorter stay.

The majority of the inpatient burden fell within the beginning of follow-up, paralleling the survival function. Among the DC patient cohort with a minimum observation time of 5 years, of the 926 patients alive at time zero, only 216 were alive five years later. The first follow-up year accounted for 30,884 inpatient days (60\% of total stay over 5 years) of which the vast majority of the burden (84.3\%) was due to liver-related admissions (Supporting Information, Table S5).

In sensitivity analysis comparing RNA-positive and RNA-negative subgroups, there was little difference in cumulative stay per patient considering admissions for any reason, except that RNAnegative patients had a slightly higher average cumulative stay for the category other liver-related compared with RNA-positive DC patients (15.5 and 13.8 days, respectively; Table S2).

\section{Proportion of admissions with liver -and non-liver related diagnoses}

Fig. 2 presents the proportion of admissions subsequent to index DC admission, according to broad hierarchically defined code category and by year of follow-up, aggregating all DC patients 
(see Supporting Information, Figs. S2 and S3 for comparable plots, but showing liver-related and non-liver related only admissions with finer-grained subcategories). Approximately $50 \%$ of all admissions within the first year following index admission were classified as HCC or DC; this proportion tended to decrease over year of follow-up.

Among the subcategories of non-liver related admissions, there was no observable pattern by year of follow-up (Fig. S3a), but the proportion of admissions classified as injuries and poisonings was highest for the youngest age-group, and the proportion of admissions in the category circulatory and respiratory tended to increase with older age-group (Fig. S3b).

\section{DISCUSSION}

The burden of hospital usage in our DC patient population is high; our DC patients spent on average 43 days in hospital (Table 2), assuming a follow-up period censored at 2 years following index DC admission. The inpatient hospital burden is greatest within the year immediately after admission for the first decompensation event, and diminishes thereafter (Fig. S1,Table S5). We observed an overall inpatient/daycase admission rate of 7.3 per person-year (12.8 admissions per person-year considering the first 6 months of follow-up).

Our estimates of the per-patient mean hospital stay varied by period of follow-up (Table S5), dropping from 33.4 days in the first year to 10.7 days in the fifth year. This finding suggests that using a single annualised value, such as that reported for DC patients (21 days) by Wright et al.,15 in cost-effectiveness analyses (CEA) could be problematic. As the Markov modelling approaches underlying CEA typically use yearly time steps, applying a single annualised length of stay to all simulated DC patient-years in the model, when the length of stay differs according to duration 
within the DC stage, may give misleading estimates of the total hospital burden borne by this patient group, and therefore could potentially influence the conclusions of the CEA. Comparison of these values is not straightforward, as Wright et al.'s values excluded patients with $<40$ days observation time, and were for 'hepatitis C-related' resource use only. Although the difference between estimates may be partly due to sample size, we believe that these difference in methodology are the most relevant.

There was a clear increasing trend in admission rate over triennium from 1999-2001; this reached 9.6 admissions per person-year in patients with first DC admission in 2011-2013. This trend probably reflects ageing of the HCV-diagnosed population, or possibly increased willingness over time to accept medical interventions. Related to this finding, there were age-group differences with respect to severity - the 60+ year age-group had the highest proportion of HCC-classified admissions, consistent with the oldest living patients having the longest duration of chronic infection.

We observed an approximate monotonic increase in annual total hospital stay over the study period, consistent with a rapid rise in the cumulative number of living DC patients over the period 1994-2013. ${ }^{3}$ The decreasing trend in mean stay for admissions for HCC or DC over the study period may indicate improved management of patients with the most severe disease, but also could reflect changing practice in outpatient vs. inpatient treatment, but we have no data to address this. The apparent decreasing trend across triennium in the prevalence of bleeding varices at first DC admission (Table 1) may be a consequence of earlier detection via screening initiatives and consequent treatment (P. Hayes, pers. comm.) 
We note that focussing on DC/HCC admissions only does not reflect the extensive hospital burden borne by this patient group. Although admissions for hepatic failure and ascites/varices led to a mean cumulative inpatient stay of 11 and 13 days, respectively (Table 2), the mean cumulative length of stay for liver-related diagnoses outwith the defined DC codeset was comparable, at 14 days. The other liver-related subcategory includes 'Alcoholic liver disease, unspecified' (K70.9) - the most frequently occurring code in this subcategory - suggesting that the high prevalence of current or historical problem alcohol use in this DC patient population contributes a significant portion of the hospitalisation burden. Furthermore, separation of RNA-positive and RNA-negative patients indicated that average cumulative stay with respect to the other liver-related category was approximately $12 \%$ longer for the latter subgroup (Table S2). This difference is consistent with a higher likelihood that progression to DC in the RNA-negative subgroup was driven more by alcohol then HCV infection.

Limitations of our study include possible misclassification error in the coding of hospital admissions. We also lacked information on clinical disease stage or on liver transplantation. The apparent greatly improved disease course in a proportion of our DC patients (as indicated by no further inpatient admissions after an initial cluster of DC and other liver-related admissions) could indicate reversal of severe disease, potentially via successful transplantation. However, relatively few liver transplantations took place during the study period, and transplant patients in Scotland can have an inpatient record generated with a code indicating prior liver transplantation; this occurred for only a small proportion. ${ }^{3}$

We considered the first 2 years following first DC admission to usefully represent the typical DC patient's inpatient history. All censoring thresholds are arbitrary, but for estimation of the burden 
of hospital usage in DC patients, using this threshold to interpret the results represents an arguably reasonable compromise between capturing the excess morbidity in this population and discounting 'background morbidity'.

In summary, we report a substantial rising burden of inpatient hospital usage among Scottish HCV-diagnosed patients with decompensated liver disease. The average length of hospital stay was clearly dependent on follow-up period, with the highest burden observed in the initial year after developing DC. During our study period there were very few DC patients who would have been initiated on direct-acting antiviral (DAA) treatment; this study should be repeated once sufficient follow-up is available for the expected increasing numbers of patients who will clear the virus following DAA treatment. Nevertheless, the present findings will be eminently useful for evaluations of the impact of interferon-free antiviral therapy in preventing DC and associated morbidity (as indicated by inpatient hospital stay), and in potentially reducing liver and non-liver related morbidity among successfully treated DC patients, for whom current guidelines recommend urgent treatment. 26

\section{ACKNOWLEDGEMENTS}

We thank the Hepatitis C Clinical Database Monitoring committee, clinical data entry staff and the following virologists for their support with the HCV diagnosis database: Dr Sheila Burns (East of Scotland Specialist Virology Centre, Royal Infirmary of Edinburgh), Dr Sheila Cameron (West of Scotland Specialist Virology Centre, Gartnavel General Hospital), Dr Paul McIntyre (Department of Medical Microbiology, Ninewells Hospital and Medical School) and Dr Pamela Molyneaux (Department of Medical Microbiology, University Medical School, Foresterhill). 


\section{REFERENCES}

(1) D'Amico G, Pasta L, Morabito A, et al. Competing risks and prognostic stages of cirrhosis: a 25year inception cohort study of 494 patients. Aliment Pharmacol Ther 2014;39:1180-1193.

(2) Fattovich G, Pantalena M, Zagni I, et al. Effect of hepatitis B and C virus infections on the natural history of compensated cirrhosis: a cohort study of 297 patients. Am J Gastroenterol 2002;97:2886-2895.

(3) McDonald S, Innes H, Aspinall E, et al. Prognosis of 1,169 hepatitis C chronically-infected patients with decompensated cirrhosis in the pre-direct acting antiviral era. J Viral Hepat 2017;24:295-303.

(4) European Association for the Study of the Liver. EASL Recommendations on Treatment of Hepatitis C 2016. J Hepatol 2017;66:153-194.

(5) Cheung MC, Walker AJ, Hudson BE, et al. Outcomes after successful direct-acting antiviral therapy for patients with chronic hepatitis C and decompensated cirrhosis. J Hepatol 2016;65:741747.

(6) Foster GR, Irving WL, Cheung MC, et al. Impact of direct acting antiviral therapy in patients with chronic hepatitis C and decompensated cirrhosis. J Hepatol 2016;64:1224-1231.

(7) el-Serag HB, Kunik M, Richardson P, Rabeneck L. Psychiatric disorders among veterans with hepatitis C infection. Gastroenterology 2002;123:476-482.

(8) Innes H, McDonald S, Hayes P, et al. Mortality in hepatitis C patients who achieve a sustained viral response compared to the general population. J Hepatol 2017;66:19-27.

(9) Petta S, Maida M, Macaluso FS, et al. Hepatitis C virus infection is associated with increased 
cardiovascular mortality: a meta-analysis of observational studies. Gastroenterology 2016;150:145155.

(10) Liu S, Cipriano LE, Holodniy M, Goldhaber-Fiebert JD. Cost-effectiveness analysis of riskfactor guided and birth-cohort screening for chronic hepatitis C infection in the United States. PLoS One 2013;8:e58975.

(11) Davis KL, Mitra D, Medjedovic J, Beam C, Rustgi V. Direct economic burden of chronic hepatitis C virus in a United States managed care population. J Clin Gastroenterol 2011;45:e17-24.

(12) McAdam-Marx C, McGarry LJ, Hane CA, Biskupiak J, Deniz B, Brixner DI. All-cause and incremental per patient per year cost associated with chronic hepatitis $C$ virus and associated liver complications in the United States: a managed care perspective. J Manag Care Pharm 2011;17:531546.

(13) Deuffic-Burban S, Schwarzinger M, Obach D, et al. Should we await IFN-free regimens to treat HCV genotype 1 treatment-naive patients? A cost-effectiveness analysis (ANRS 95141). J Hepatol 2014;61:7-14.

(14) van Santen DK, de Vos AS, Matser A, et al. Cost-effectiveness of hepatitis C treatment for people who inject drugs and the impact of the type of epidemic: Extrapolating from Amsterdam, the Netherlands. PLoS One 2016;11:e0163488.

(15) Wright M, Grieve R, Roberts J, Main J, Thomas HC, UK Mild Hepatitis C Trial Investigators. Health benefits of antiviral therapy for mild chronic hepatitis C: randomised controlled trial and economic evaluation. Health Technol Assess 2006;10:1-113, iii.

(16) Wright M, Forton D, Main J, et al. Treatment of histologically mild hepatitis C virus infection with interferon and ribavirin: a multicentre randomized controlled trial. J Viral Hepat 2005;12:58- 
66.

(17) Martin NK, Vickerman P, Dore GJ, et al. Prioritization of HCV treatment in the direct-acting antiviral era: An economic evaluation. J Hepatol 2016;65:17-25.

(18) Scott N, Iser DM, Thompson AJ, Doyle JS, Hellard ME. Cost-effectiveness of treating chronic hepatitis $\mathrm{C}$ virus with direct-acting antivirals in people who inject drugs in Australia. J Gastroenterol Hepatol 2016;31:872-882.

(19) Kendrick S, Clarke J. The Scottish Record Linkage System. Health Bulletin (Edinburgh) $1993 ; 51: 72-79$.

(20) Shaw L, Taylor A, Roy K, et al. Establishment of a database of diagnosed HCV-infected persons in Scotland. Commun Dis Public Health 2003;6:305-310.

(21) Health Protection Scotland. Surveillance of known hepatitis C antibody positive cases in Scotland: Results to 31 December 2013. HPS Weekly Report: Health Protection Scotland 2014;18(2011/18):243-249.

(22) Costella A, Goldberg D, Harris H, et al. Hepatitis C in the UK 2015 Report. London: Public Health England; 2015.

(23) Innes HA, McDonald SA, Dillon JF, et al. Toward a more complete understanding of the association between a hepatitis $C$ sustained viral response and cause-specific outcomes. Hepatology 2015;62:355-364.

(24) Ioannou GN, Bryson CL, Weiss NS, Miller R, Scott JD, Boyko EJ. The prevalence of cirrhosis and hepatocellular carcinoma in patients with human immunodeficiency virus infection. Hepatology 2013;57:249-257. 
(25) R Development Core Team. R: A language and environment for statistical computing. Vienna, Austria; 2014.

(26) European Association for Study of Liver. EASL Clinical Practice Guidelines: management of hepatitis C virus infection. J Hepatol 2014;60:392-420. 
Table 1. (a) Characteristics of, and (b) total inpatient admissions among HCV-diagnosed patients with decompensated cirrhosis (DC), by triennium of first DC admission; study population is defined as persons with a first hospital admission with mention of DC within the period 1996-2013 $(n=1,543)$.

Period of first DC admission

$1996-1998 \quad 1999-2001 \quad 2002-2004 \quad 2005-2007 \quad 2008-2010 \quad 2011-2013 \quad$ Total

(a) Patient characteristics

$\begin{array}{llllllll}N \text { patients } & 107 & 164 & 217 & 279 & 363 & 413 & 1543 \\ \text { Males, } n(\%) & 78(72.9) & 119(72.6) & 164(75.6) & 203(72.8) & 263(72.5) & 302(73.1) & 1129(73.2) \\ \text { Mean age in } & & & & & & & \\ \text { years (SD) } & 46.7(14.8) & 48.2(13.9) & 46.7(12.1) & 45.2(10.1) & 47.2(10.8) & 48.2(10.3) & 47.1 \\ (11.5) & & & & & & & \end{array}$

Risk group

$\begin{array}{lccccccc}\text { PWID, } n(\%) & 41(38.3) & 49(29.9) & 101(46.5) & 156(55.9) & 164(45.2) & 199(48.2) & 710(46.0) \\ \text { NonPWID, } n(\%) & 12(11.2) & 20(12.2) & 16(7.4) & 21(7.5) & 30(8.3) & 19(4.6) & 118(7.6) \\ \text { NK, } n(\%) & 54(50.5) & 95(57.9) & 100(46.1) & 102(36.6) & 169(46.6) & 195(47.2) & 715(46.3)\end{array}$

Prevalence of DC complications at first DC admission (\%)

$\begin{array}{llllllll}\text { Ascites } & 43 & 48 & 57 & 59 & 61 & 61 & 57.4 \\ \text { Bleeding varices } & 22 & 21 & 14 & 13 & 12 & 12 & 13.9 \\ \begin{array}{l}\text { Hepatic failure, } \\ \text { incl. enceph. }\end{array} & 29 & 24 & 20 & 18 & 19 & 20 & 20.5\end{array}$

Alcoholic hepatic

$\begin{array}{llllllll}\text { failure } & 9 & 12 & 12 & 14 & 12 & 14 & 12.5 \\ \text { Hepatorenal } & & & & & & & \end{array}$

$\begin{array}{llllllll}\text { syndrome } & 4 & 3 & 7 & 3 & 4 & 2 & 3.5\end{array}$

Prior alcohol-related

admission (\%)

45

$52 \quad 53$

57

63

56

56.3

Prior admission

for HCC (\%)

$6 \quad 4$

2

3

4

4.2

HCV RNA status at first DC admission (\%)

$\begin{array}{lrrrrrrr}\text { RNA +ve } & 47.7 & 61.6 & 75.6 & 81.7 & 82.1 & 84.5 & 77.2 \\ \text { RNA -ve } & 6.5 & 11.0 & 13.8 & 13.6 & 13.5 & 12.6 & 12.6 \\ \text { NK } & 45.8 & 27.4 & 10.6 & 4.7 & 4.4 & 2.9 & 10.2\end{array}$

Dead as of

31 May 2014

96

135

179

218

260

198

1086 
(b) Inpatient admissions

\begin{tabular}{|c|c|c|c|c|c|c|c|}
\hline $\mathrm{N}$ patients & 107 & 164 & 217 & 279 & 363 & 413 & 1543 \\
\hline Total admissions* & 532 & 807 & 1332 & 1887 & 2565 & 3056 & 10179 \\
\hline Person-years* & 72 & 136 & 198 & 259 & 308 & 319 & 1292 \\
\hline \multicolumn{8}{|l|}{ Admissions per } \\
\hline person-year* & 7.42 & 5.92 & 6.74 & 7.30 & 8.32 & 9.57 & 7.88 \\
\hline \multicolumn{8}{|l|}{ Median stay per } \\
\hline admission (days) [IQR] & 3 [1-9] & 3 [1-9] & $3[1-8]$ & 3 [1-7] & 3 [1-7] & 2 [1-5] & $3[1-7]$ \\
\hline
\end{tabular}

Note: PWID = people who inject drugs; $\mathrm{NK}=$ not known; diagnosis codes: ascites $=$ R18; bleeding varices = I85.0, I98.3; hepatic failure $=\mathrm{K} 72.1, \mathrm{~K} 72.9$; alcoholic hepatic failure $=\mathrm{K} 70.4$; hepatorenal syndrome $=\mathrm{K} 76.7$; $\mathrm{IQR}=$ interquartile range. Median length of stay is per admission.

* Based on an allotted additional 5 months of follow-up following each 3-year period, to ensure comparability in admission rates across period of first DC admission. 
Table 2. Inpatient hospital usage by HCV-diagnosed patients first admitted for decompensated cirrhosis (DC) in the period 1 January 1996 to 31 May $2012(n=1312)$, according to different followup periods (entire, first 2 years, first year, and first 6 months). All hospital admissions during patient's entire history until censoring boundary, starting with first DC admission, are presented, and are additionally stratified by hierarchically-defined diagnosis code category.

\begin{tabular}{lcccc} 
& Entire period & First 2 years & First year & First 6 months \\
\hline Total admissions & 13863 & 9102 & 6786 & 4823 \\
$\begin{array}{l}\text { Person-years } \\
\begin{array}{l}\text { Admissions per } \\
\text { person-year }\end{array}\end{array}$ & 3036 & 1247 & 708 & 376 \\
$\begin{array}{l}\text { Median length of stay } \\
\text { per admission (days) }[\mathrm{IQR}]\end{array}$ & 4.57 & 7.30 & 9.59 & 12.81 \\
\end{tabular}

Mean cumulative length of stay per patient (days) according to admission code category:

$\begin{array}{lrrrr}\text { All } & 59.8 & 43.3 & 34.2 & 26.1 \\ \text { Liver-related } & 50.6 & 38.8 & 31.1 & 24.0 \\ \text { HCC } & 22.3 & 1.6 & 1.4 & 1.1 \\ \text { DC - hepatic failure } & 24.9 & 10.8 & 9.1 & 7.3 \\ \text { DC - ascites/varices } & 22.1 & 12.7 & 11.4 & 10.0 \\ \text { Other liver-related } & 33.4 & 13.6 & 9.3 & 5.6 \\ \text { Non-liver related only } & 20.2 & 4.5 & 3.1 & 2.1 \\ \end{array}$

Note: $\mathrm{IQR}=$ interquartile range. Median length of stay is per admission; mean cumulative length of stay is total stay (summing multiple admissions) within follow-up period summed over all patients, then divided by the number of patients. 
Table 3. Results of multivariate Poisson regression analysis of patient and other factors associated with cumulative length of stay, among HCV-diagnosed patients first admitted for decompensated cirrhosis in period 1996-2013. Follow-up is censored at 2 years following first DC admission.

Adjusted rate ratio $95 \%$ confidence interval

\begin{tabular}{|c|c|c|}
\hline Male sex & 0.81 & $0.80-0.83$ \\
\hline \multicolumn{3}{|l|}{ Age at first DC admission } \\
\hline$<40$ years & 0.94 & $0.92-0.96$ \\
\hline $40-49$ years & Ref. & \\
\hline $50-59$ years & 1.01 & $0.99-1.04$ \\
\hline $60+$ years & 1.11 & $1.08-1.14$ \\
\hline \multicolumn{3}{|l|}{ Risk group } \\
\hline PWID & Ref. & \\
\hline NonPWID, & 1.01 & $0.97-1.04$ \\
\hline NK & 1.00 & $0.98-1.02$ \\
\hline Prior admission for HCC & 0.68 & $0.64-0.71$ \\
\hline Prior alcohol-related admission & 1.21 & $1.19-1.23$ \\
\hline \multicolumn{3}{|c|}{ HCV RNA status at first DC admission } \\
\hline RNA +ve & Ref. & \\
\hline RNA -ve & 1.00 & $0.98-1.03$ \\
\hline NK & 1.00 & $0.97-1.03$ \\
\hline \multicolumn{3}{|c|}{ Hierarchically-defined diagnosis code category, first DC admission } \\
\hline Hepatic failure & Ref. & \\
\hline Ascites & 1.12 & $1.10-1.15$ \\
\hline Bleeding varices & 0.83 & $0.80-0.85$ \\
\hline \multicolumn{3}{|l|}{ Period of first DC admission } \\
\hline 1996-1998 & 0.96 & $0.92-1.00$ \\
\hline 1999-2001 & 0.94 & $0.91-0.97$ \\
\hline 2002-2004 & 1.17 & $1.13-1.21$ \\
\hline 2005-2007 & 1.12 & $1.09-1.15$ \\
\hline 2008-2010 & 1.06 & 1.03-1.09 \\
\hline 2011-2013 & Ref. & \\
\hline
\end{tabular}




\section{FIGURE LEGENDS}

Fig. 1. Burden of inpatient hospital usage among HCV-diagnosed DC patients in the period 1996-2013. Total stay in hospital is plotted by calendar year and stratified by hierarchically-defined broad diagnosis code category. The table below the $\mathrm{x}$-axis indicate the annual total number of admissions.

Fig. 2. Distribution over inpatient admission diagnosis category among HCV-diagnosed persons (first admitted with DC in period 1996-2013). Distribution of all recorded hospital admissions subsequent to patient's first DC admission among the set of three hierarchicallydefined diagnosis code categories is plotted by time since first DC admission. The table below the $\mathrm{x}$-axis indicate annual total numbers of admissions contributing to each stacked bar. The $\mathrm{x}$-axis label ' 0 ' relates to $[0,1) ;$ ' 1 ' relates to $[1,2)$, etc. 